\title{
The Virgo Coating Collaboration: a detailed study on thermoelasticity in crystalline materials and other research lines
}

\author{
E. Cesarini ${ }^{* a}$, M. Lorenzini ${ }^{b}$, A. Amato ${ }^{c}$, G. Cagnoli ${ }^{c, d}$, Q. Cassar ${ }^{c}$, J. Dickmann ${ }^{e}$, \\ M. Granata ${ }^{c}$, V. Fafone ${ }^{f, g}$, D. Heinert ${ }^{h}$, S. Kroker ${ }^{i, e}$, D. Lumaca ${ }^{f, g}$, F. Martelli ${ }^{j}$, \\ L.O. Mereni ${ }^{c}$, R. Nawrodt ${ }^{h}$, F. Piergiovanni ${ }^{j}$, C. B. Rojas Hurtado ${ }^{e}$ \\ ${ }^{a}$ Centro Studi e Ricerche Enrico Fermi, I-00184 Roma, Italy \\ ${ }^{b}$ Gran Sasso Science Institute, I-67100, L'Aquila Italy \\ ${ }^{c}$ Laboratoire des Matériaux Avancés (LMA), CNRS/IN2P3, F-69622 Villeurbanne, France \\ ${ }^{d}$ Université Claude Bernard Lyon 1, F-69622 Villeurbanne, France \\ ${ }^{e}$ Technical University of Braunschweig, LENA Laboratory for Emerging Nanometrolog, \\ Pockelsstraße 14, 38106 Braunschweig, Germany \\ ${ }^{f}$ Università di Roma Tor Vergata, I-00133 Roma, Italy \\ ${ }^{g}$ INFN, Sezione di Roma Tor Vergata, I-00133 Roma, Italy \\ ${ }^{h}$ Institut für Festkörperphysik, Friedrich-Schiller-Universität Jena, Helmholtzweg 5, D-07743 \\ Jena, Germany \\ ${ }^{i}$ Physikalisch-Technische Bundesanstalt, Bundesallee 100, 38116 Braunschweig, Germany \\ j'Università degli Studi di Urbino 'Carlo Bo', I-61029 Urbino, Italy \\ E-mail: elisabetta.cesarinieroma2.infn.it
}

The visibility distance of interferometric gravitational wave detectors is limited by mirror thermal noise at mid-range frequency, where the first coalescence GW signals have been detected and where many others are expected in the next future. In particular, for enhanced second generation and third generation we need to increase the performance of the test mass multilayer reflective coatings. The Virgo collaboration is setting up a coating R\&D group on many issues, including especially metrology (loss angle measurements, thermoelastic effect modeling), new materials (new oxides, nitrates, fluoride, new cosputtered mixing and nanolayered composites) completely characterized (optically, mechanically and morphologically), optimization of deposition parameters, with the aim of developing new coating materials and technologies for the AdVirgo upgrades and for future detectors. Another objective is to understand the losses in amorphous materials, framing coating research in the more general context of the physics of glasses and amorphous materials. One of the developed research lines is the study of thermoelastic damping in those crystalline materials that are promising candidates for cryogenic test masses and particularly suitable substrates for coating research. A detailed discussion on models, based on a semi-analytical calculation starting from the heat diffusion equation, for crystalline substrates will be reported. A new study on the changes in thermoelastic loss after coating deposition, with some preliminary results, will be also reported.

GRAvitational-waves Science\& technology Symposium - GRASS2018

1-2 March 2018

Palazzo Moroni, Padova (Italy)

${ }^{*}$ Speaker. 


\section{Introduction}

The coincident detection of a gravitational wave (GW) emitted by two coalescing black holes (BH) by the two LIGO detectors in USA on September 15th 2015, initiated the era of GW astronomy [1]. This first event was followed by other five detections of binary $\mathrm{BH}(\mathrm{BBH})$ mergers. On August 14th 2017, a BBH coalescence was observed by LIGO and Virgo. With the Advanced Virgo (AdV) detector coming into operation, distance measurement and sky localization significantly improved from hundreds to tens of square degrees [2]. The efforts of this joint observing program allowed, on August 17th 2017, the first observation of the GW signal GW170817 from a binary neutron stars coalescence [3]. This event was also responsible for the electromagnetic (EM) emission observed 1.7 second after GW170817 (gamma-ray burst GRB170817A). The presence of AdV again dramatically improved the localization capabilities of the GW network and enabled EM partners to quickly identify a counterpart in the galaxy NGC4993 [4]. The continuing efforts to enhance the sensitivity of the detectors along with longer future observing runs will deliver greater numbers of detections at lower noise levels, entering the pathway to a widely opened GW astronomy. For the current and future interferometers (ITF), in the middle frequency band, the sensitivity is limited by mirror coating thermal noise, generated by the internal friction in multilayer dielectric coatings [5]. Advanced detector upgrades are planned to improve the sensitivity and the event rates within 2020. The study of thermal noise in multilayer coatings has been driven also by interests in other fields, for example, precision measurement, semiconductor industry needs and other cutting edge developments.

\section{The Virgo coating $R \& D$ collaboration}

In the last decade, the path toward lower noise coatings has begun to get clearer, thanks to national and international research projects. Theoretical guidance and technological expertise have also grown. Molecular dynamics simulations give insight in the nature of the noise sources, the synthesis processes and experimental characterization methods are being clarified. The time is now ripe for an integrated effort coordinating the results of previous research and setting the stage for a structured plan. The Virgo coating R\&D collaboration is made up of many research groups of the Virgo collaboration and has within it coating deposition technologies, internal friction measurements facilities, optical and structural characterization facilities. Furthermore, the members of this collaboration have developed the nowadays employed mutilayer reflective coatings and they gained the proper know-out in terms of thermal noise studies and material characterization, having a leading role in the world research on low-noise coatings. This group works on many subjects, especially metrology (mechanical characterization, thermo-elasticity calculation) new coating materials (new oxides, nitrates, fluoride, new mixing and nano-layered composites), deposition parameters, with the aim of developing new multilayer reflective coatings for the AdVirgo upgrades and new technologies for future detectors. In this work the thermo-elasticity calculation for crystalline materials before and after coating deposition is reported.

\section{Dissipation measurements in thin film with GeNS}

The advanced ground based detectors of gravitational waves (GW) are Michelson ITFs with 
arms of few kilometres and with suspended, "free falling" mirrors that act as test masses. They behave as transducers to convert the space-time strain perturbation caused by a passing GW to a measurable phase lag of laser light. A displacement of the mirror face is associated via the Fluctuation-Dissipation theorem with the mechanical losses in the mirror materials [6]. Because of the low elastic dissipation of the mirror bulk material, the internal friction in the multilayer dielectric coatings is dominant. Dissipation can be described in terms of a loss angle $\phi$, that is, the phase of the material's complex Young's modulus, $Y$. The power spectral density (PSD) of the coating Brownian noise is related to the effective coating loss angle $\phi_{c}$, according to Levin [7], by:

$$
S_{B}(f)=\frac{2 k_{B} T\left(1-\sigma_{s}^{2}\right)}{T^{3 / 2} f w Y_{S}} \phi_{C},
$$

where $k_{B}$ is Boltzmann's constant, $T$ the absolute temperature, $w$ the effective laser Gaussian beam radius constrained by optical interferometer configuration, $\sigma_{s}$ the Poisson's ratio of the substrate, and $Y_{s}$ its Young's modulus. Working at room temperature with fixed beam size, decreasing the thermal noise level means reducing the coating loss angle. When the losses become extremely low, as in the case of the materials used in GW detectors, their measurement becomes very challenging and a profound knowledge of various loss mechanisms is essential. A key in the coating research is the method to work out the dissipation value of the material deposited. The Virgo collaboration developed a nodal suspension called GeNS that can provide a reliable measurement of this parameter [8]. In GeNS a disk shaped sample is placed in equilibrium on the top of a sphere. Selecting modes with nodes at the support point makes suspension losses negligible (see fig.1). GeNS measurements are very rapid and obtain a reproducibility of 5\%, much better than any other methods currently available. GeNS is aknowledged as one of the most powerful tools for mechanical loss characterization [9],[10]. The GeNS suspension uses a ring-down technique where the free decay

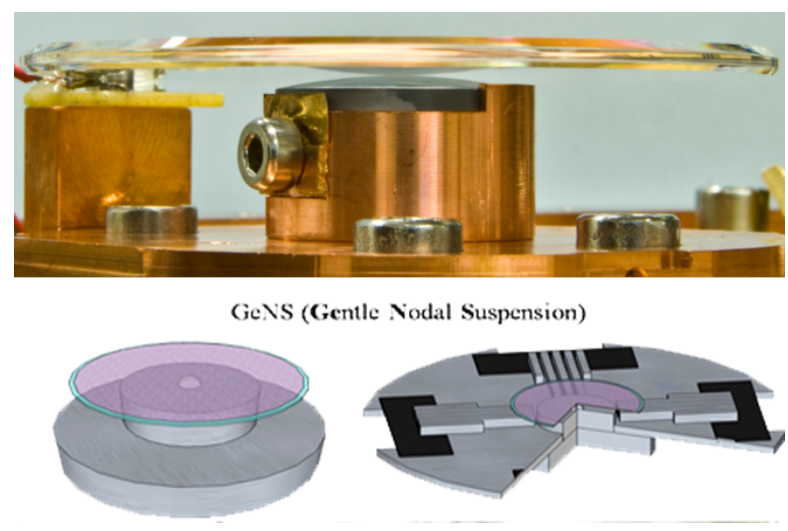

Figure 1: A picture and a schematic view of the GeNS suspension

time is measured with a displacement sensor (optical lever or capacitor sensor). From the decay time $\tau$ we can work out the loss angle for every resonant mode through: $\phi_{i}=\left(\pi f_{i} \tau\right)^{-1}$. In order to simplify the interpretation of the results, a FEA allows to compute the expected resonant frequencies of the modes (usually with an error with respect to the measured value smaller than $1 \%$ ) as well as the shape of their vibration. Thus the measured frequencies and loss angles can be associated to a mode order $(\mathrm{n}, \mathrm{m})$, where $\mathrm{n}$ and $\mathrm{m}$ are the number of azimuthal and radial nodes, respectively. 


\section{Thermoelastic damping calculation}

Future gravitational wave detectors are proposed to be operated at cryogenic temperatures. For these cryogenic detectors promising materials are crystalline silicon or sapphire, as well. Coating losses are evaluated looking at their effect on the substrate loss. Substrate is measured before coating deposition and substrate losses are supposed independent by the coating deposition process. To this end, the substrate loss is measured. After that, energy stored in the coating can be evaluated by FEM, analytically or with a direct measurement. For reliable information on the coating losses, the knowledge of the substrate losses is mandatory. In thin silicon or sapphire discs, at acoustic frequencies, the main contribution to the substrate loss is expected to be due to thermoelastic loss. The thermoelastic effect is a relaxation process caused by the heat exchanged within the vibrating body, between compressed and expanded regions. Independently of the pursued approach, the explicit computation of the thermoelastic loss for a body of general shape or structure is overwhelmingly complicate; however, it can be highly simplified by taking into account geometrical symmetries. Zener solved the problem for a beam, in which two dimensions are neglected with respect to the beam length [11]. Cagnoli et al. [12] presented results valid for a cylinder, that eventually assume a simpler form for a thin disc in which one of the three dimensions is negligible. The approach to compute the thermoelastic loss follows the derivation outlined by Zener, based on the evaluation of entropy variation. The energy dissipated per cycle is related to the temperature field through entropy:

$$
\Delta E=T_{0} \Delta S=\int_{V} \oint \frac{k}{T_{0}}(\nabla T)^{2} d t d V .
$$

The final formula for the thermoelastic loss turns out to be very simple, it is obtained as a single Debye's peak with peak angular frequency:

$$
\omega_{\text {peak }}=\frac{k}{c_{V}}\left(\frac{\pi}{L}\right)^{2}
$$

where $k$ is the thermal conductivity, $c_{V}$ is the heat capacity, $L$ is the disc thickness. This result coincides with the one obtained by Zener in his original work on rectangular reeds, and weighted by a dilution factor $D_{d i l}$ defined as the ratio between the pure dilation energy and the total elastic energy:

$$
\phi_{D t h, e l}=D_{d i l} \frac{(3 \alpha)^{2} K T_{0}}{c_{V}\left(\omega^{2}+\omega_{\text {peak }}^{2}\right)},
$$

where $\alpha$ is the thermal expansion and $K$ is the bulk modulus. For crystalline materials, cubic as silicon and trigonal as sapphire, the ratio $\left(D_{d i l}\right)$ is influenced by the mode shape, so the thermoelastic loss will show a branching behaviour, depending on mode families.

\subsection{Silicon}

For thin silicon discs the $D_{\text {dil }}$ dilution factor can be extracted through FEA. We measured CZ-grown p-type (100) boron-doped silicon wafers having a resistivity from $0.1 \Omega \mathrm{cm}$ to $1 \Omega \mathrm{cm}$, provided by Sil'Tronix-ST. These wafers have a nominal diameter of $76.2 \mathrm{~mm}$ and a varying thickness of about 430 to $470 \mu \mathrm{m}$. We performed FEA to work out $D_{d i l}$ and we compared measurements with calculation, obtaining a good agreement (see fig.2) within the measurement error with GeNS 
( $<10 \%, 5 \%$ typical). Uncertainty in the evaluation of equation 4.3 is of the same order, coming mainly from the error in the measurement of the thickness $L$. For a few modes, the measured loss is largely higher than the one computed with our model; in all these cases, the disc centre is found by FEA analysis to be no longer a nodal point due to the anistropic nature of the material.
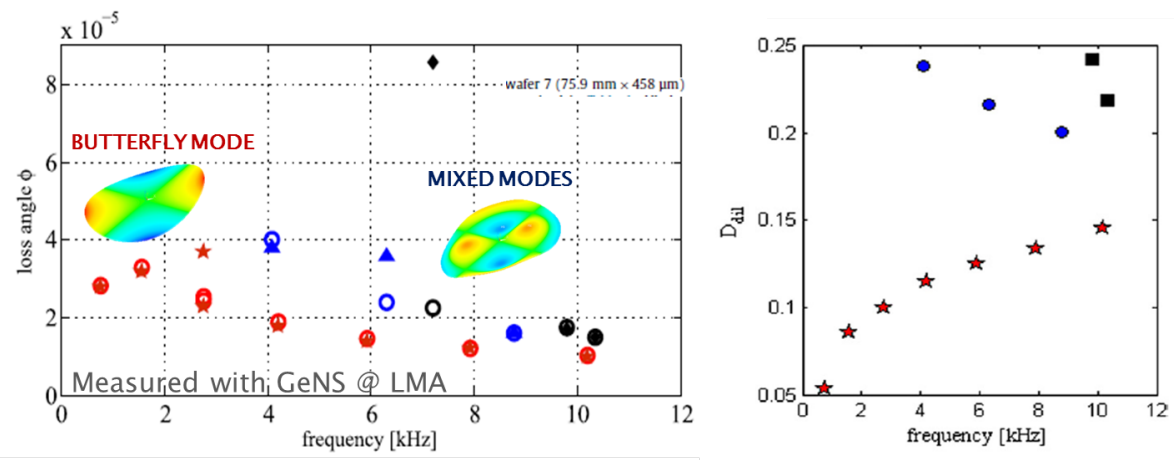

Figure 2: left. Measured loss angle values compared with calculated loss angle values for a silicon wafer of $458 \mu \mathrm{m}$ thickness. Measured values are reported according to different colors for different mode shape families, red stars for $(n, 0)$ modes, blue triangles for $(n, 1)$ modes and black diamonds for $(n, 2)$ modes. Computed values are represented by red, blue and black circles. right. $D_{d i l}$ evaluated by FEA for the first 13 resonant modes, branched according to the red, blue and black mode families.

\subsection{Sapphire}

For thin sapphire discs we compared experimental data with thermoelastic damping calculation with the same formula used for silicon wafers. $D_{\text {dil }}$ has been worked out through FEA simulation, using two different tools Ansys and Comsol. We used a sapphire sample 2 inches diameter and $1 \mathrm{~mm}$ thickness discs, double-sided polished, with crystal orientation: <0001>0.25 deg misoriented. Also for this crystal we obtained a good agreement (see fig.3). Here again, the considerations on uncertainty made for the silicon disc in the previous paragraph are valid. A direct computation of the thermoelastic loss with Comsol is also shown.

\section{Thermoelasticity after coating deposition}

It has been observed that depositing a $\mathrm{SiO}_{2}$ film on both faces on a silicon disc affects the thermoelastic contribution to mechanical loss. See as example figure 4, where the losses are globally reduced after coating deposition: given that the coating layer is expected to spoil the mechanical quality, a different thermoelastic loss in the bulk is needed to explain the results. Bishop and Kinra in 1996 showed that the total effect of a coating deposition in laminated micromechanical beam resonator on the thermoelastic loss angle is influenced mainly by changing in thickness, heat capacity and thermal expansion coefficient, while the thermal conductivity has no great effect [13]. We used the same model applying it to our silicon disc sample (diameter 3 inches of and thickness of $468 \mu$ $\mathrm{m}$ ) and considering a $\mathrm{SiO}_{2}$ film of $2.2 \mu \mathrm{m}$ on both faces. We try to reproduce the thermoelastic loss of the bare substrates before and after the $\mathrm{SiO}_{2}$ coating deposition (see fig. 5), finding a variation of 


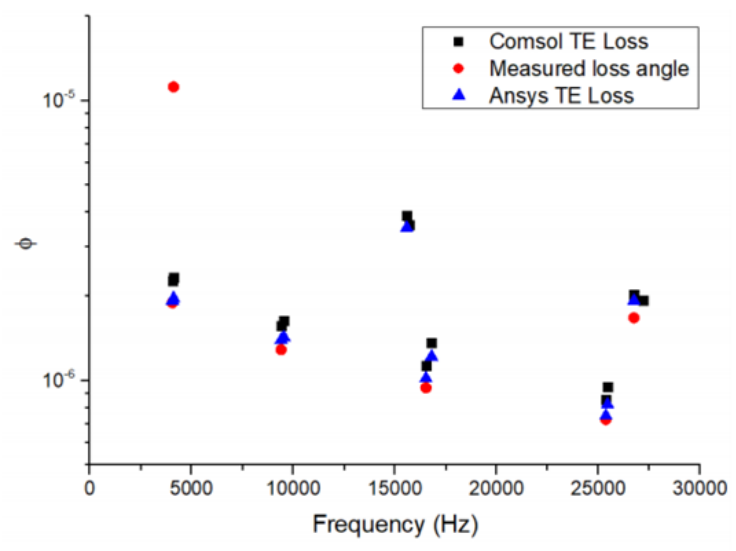

Figure 3: Measured loss angle (red circles) compared with calculated loss angle (blue triangles) for a sapphire disc of $1 \mathrm{~mm}$ thickness. Thermoelastic loss obtained with Comsol FEA is also shown (black squares).

the loss angles roughly of the same order of what was measured. The approach seems to be promising and it will be investigated in deeper detail. It is important to take this effects into account when we try to work out the coating loss. This allows us to use crystalline substrates for coating research, because we can substract their modeled contribution. It is important to use crystalline materials not only because they are interesting for third generation of GW detectors working at cryogenic temperature but also because the knowledge of mechanical properties at low temperatures is strongly required to predict their dissipation mechanism and the thermal noise limited sensitivity.

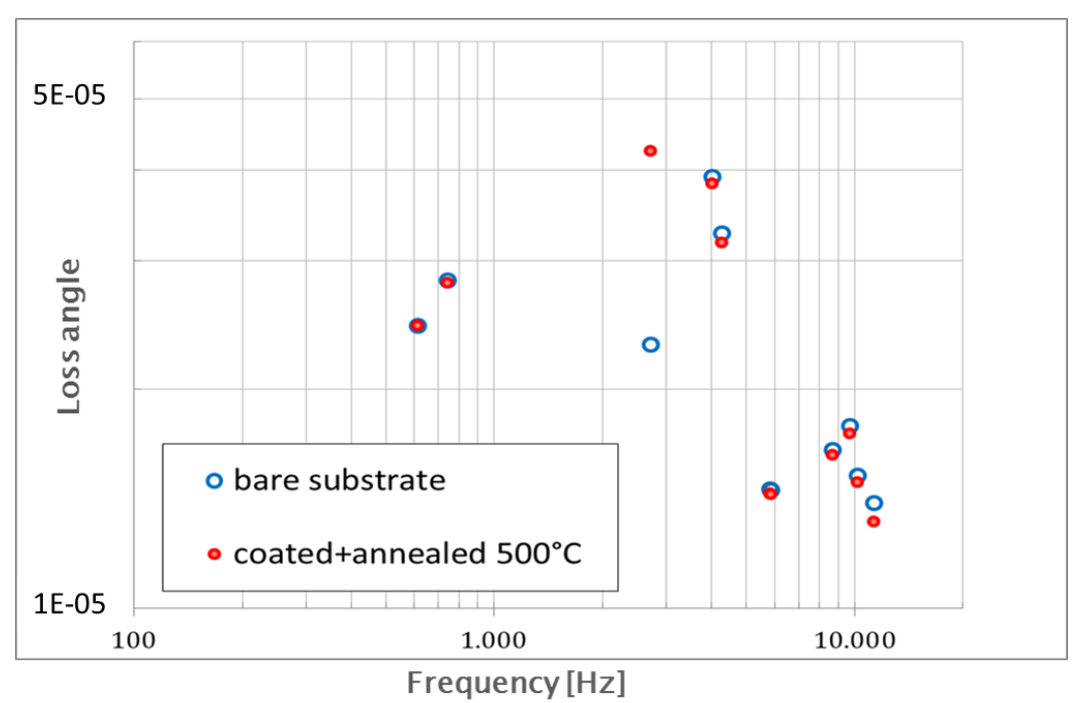

Figure 4: Measured loss angle of a bare silicon substrates and coated silicon substrate. The deposited coating is a $\mathrm{SiO}_{2}$ film $2.2 \mu \mathrm{m}$ thick on both faces. Mesurement error $(\simeq 5 \%)$ is not reported to avoid spoiling the plot readability. 


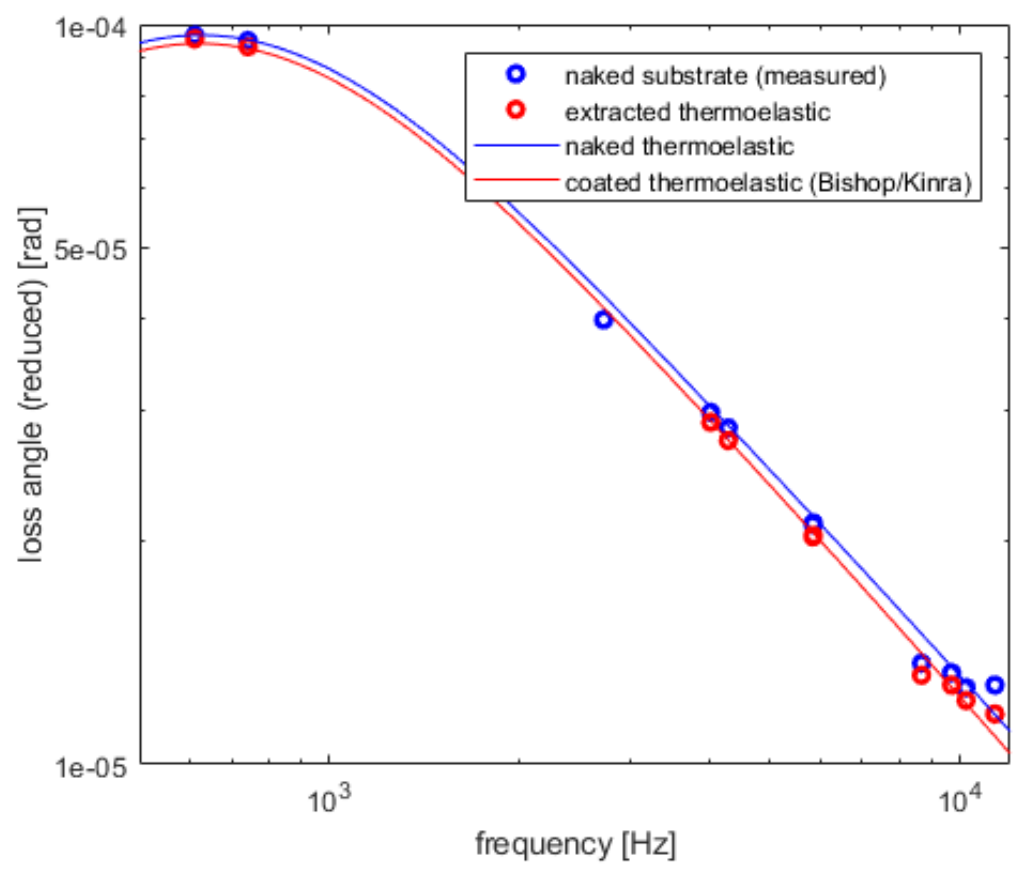

Figure 5: Measured reduced loss angle compared with calculation from Bishop and Kinra model for a silicon substrate before and after the coating deposition. The reduced loss angle is the value of the loss angle divided by $D_{d i l}$.

\section{Conclusions}

The Virgo coating R\&D collaboration aims at developing new multilayer reflective coatings for the AdVirgo upgrades and new technologies for future detectors. The work of the collaboration includes metrology and investigations on new coating materials or techniques, approaching the understanding of the losses in amorphous materials in the general frame of physics of glasses. As part of this work, the study of dissipation in discs is fundamental to correctly extract the value of coating loss from measurements. We described a semi-analytical method to compute the thermoelastic mechanical loss in silicon and sapphire discs, comparing it with experimental findings and showing that this is indeed the main contribution to the loss angle in bare discs to be used with GeNS for the measurement of coating losses. Thermoelastic loss is also affected by coating deposition, and we have shown that an approach based on the model of Bishop and Kinra is promising in accounting for the effect. According to our results, the loss of films deposited on silicon or sapphire discs can be accurately measured, provided that their effect on loss angle is comparatively larger than the GeNS measurement error.

\section{References}

[1] B.P. Abbot et al. (LIGO Scientific Collaboration and Virgo Collaboration), Observation of Gravitational Waves from a Binary Black Hole Merger, Phys. Rev. Lett. 116 (2016) 
[2] B.P. Abbot et al. (LIGO Scientific Collaboration and Virgo Collaboration), GW170814: A Three-Detector Observation of Gravitational Waves from a Binary Black Hole Coalescence, Phys. Rev. Lett. 119 (2017)

[3] B.P. Abbot et al. (LIGO Scientific Collaboration and Virgo Collaboration), GW170817: Observation of Gravitational Waves from a Binary Neutron Star Inspiral, Phys. Rev. Lett. 119 (2017)

[4] LIGO Scientific Collaboration, Virgo Collaboration, Fermi Gamma-Ray Burst Monitor, INTEGRAL Gravitational Waves and Gamma-rays from a Binary Neutron Star Merger: GW170817 and GRB 170817A, The Astrophysical Journal Letters 848:L13 (2017), arXiv:1710.05834

[5] S. D. Penn et al. Mechanical Loss in Tantala/Silica Dielectric Mirror Coatings Class. Quantum Grav. 20 (2003)

[6] H.B. Callen and T.A. Welton Irreversibility and Generalized Noise,Phys. Rev. 83(1951)

[7] Y. Levin, Internal thermal noise in the LIGO test masses: A direct approach, Phys. Rev. D 57 (1998)

[8] E. Cesarini et al., A "gentle" nodal suspension for measurements of the acoustic attenuation in materials, Rev.Sci. Instrum. 80 (2009)

[9] M. Granata et al., Internal Friction and Young's Modulus Measurements on $\mathrm{SiO}_{2}$ and $\mathrm{Ta}_{2} \mathrm{O}_{5}$ Films Done with an Ultra-High Q Silicon-Wafer Suspension, Arch. Metall. Mater. 60(1) (2015)

[10] G. Vajente et al., A high throughput instrument to measure mechanical losses in thin film coatings, Rev. Sci. Instrum. 88 (2017)

[11] C. Zener, Internal Friction in Solids. I. Theory of Internal Friction in Reeds, Phys. Rev. 52 (1937)

[12] G. Cagnoli, et al., Mode-dependent mechanical losses in disc resonators, Phys. Lett. A 5 (2017)

[13] J. E. Bishop and V. K. Kinra, Equivalece of the mechanical and entropic descriptions of elastothermodynamic damping in composites materials, Mech. Compos. Mater. Struct. 3 (1996) 